\title{
Caracterización estructural y funcional de los macroinvertebrados en los bañados de desborde fluvial del área pampeana
}

\author{
Alberto Rodrigues Capítulo ${ }^{1,2,3}$, Laura Armendáriz ${ }^{1,3}$, Augusto SirI 1,2,3, Paula Altierl ${ }^{1,2}$, \\ Carolina Ocon ${ }^{1,2,3}$, Bianca Cortese ${ }^{2,4}$, Ludmila Rodríguez Catanzaro ${ }^{1,2}$, Juan Pablo \\ Zanotto Arpellino ${ }^{1,2}$, Marianela Rodríguez ${ }^{1,2}$ y Mariano Donato M, 2,3 $^{2}$ \\ 1'Instituto de Limnología “Dr. Raúl A. Ringuelet" (CONICET - UNLP), La Plata, Argentina \\ ${ }^{2}$ Consejo Nacional de Investigaciones Científicas Técnicas (CONICET) \\ ${ }^{3}$ Facultad de Ciencias Naturales y Museo, UNLP \\ ${ }^{4}$ Centro de Investigación Esquel de Montaña y Estepa Patagónica (CIEMEP), CONICET - UNPSJB, Esquel, \\ Argentina.
}

E-mail: acapitul@ilpla.edu.ar

RESUMEN. En este estudio se caracteriza el ensamble de macroinvertebrados en bañados de desborde fluvial de cuatro arroyos pampeanos bonaerenses con diferentes actividades en el uso del suelo (intensa horticultura y ganadería extensiva) desde un punto de vista estructural y funcional. Se aplicaron índices bióticos, ecológicos, análisis multivariados y se analizaron los ensambles de oligoquetos y quironómidos. Los mayores valores diversidad y riqueza taxonómica de macroinvertebrados correspondieron al bañado de Chubichaminí $\left(H^{\prime}=1,9 ; S=19,25\right)$. Este arroyo presentó aguas ligeramente poluídas, siendo aceptable en términos de calidad del agua. El análisis multivariado ordenó a los sitios de los bañados Del Gato y Carnaval con la mayor carga de nutrientes vinculados a los taxa más tolerantes como los dípteros Stratiomyidae, Ephydridae y nematodes, oligoquetos y gasterópodos Physidae. La mayor diversidad y riqueza de oligoquetos se registró en los bañados del Cajaravillas y Chubichaminí en los meses cálidos y las diferencias encontradas se debieron principalmente a Limnodrilus hoffmeisteri Claparède, 1862. El ensamble de quironómidos estuvo conformado por 24 taxones pertenecientes a Chironominae, Orthocladiinae y Tanypodinae. El género Chironomus Meigen fue más abundante en el $A^{\circ}$ Del Gato, correspondiendo principalmente a C. calligraphus Goeldi, asociado a ambientes con evidente impacto antrópico. Los sitios estudiados mostraron diferentes patrones en la proporción de grupos funcionales alimentarios sin una clara relación con el uso del suelo. Los índices aplicados en el ensamble de macroinvertebrados resultaron efectivos para evaluar el estado ecológico de los bañados analizados.

Palabras clave: Índices bióticos, grupos funcionales alimentarios, macroinvertebrados bentónicos, oligoquetos, quironómidos.

ABSTRACT. In this study, the assemblage of aquatic macroinvertebrates in the riverine wetlands (RW) of four streams in the Pampean region of Buenos Aires with different activities in land use (intensive horticulture and extensive livestock) is characterized from a structural and functional point of view. Biotic and ecological indices and multivariate analyses were applied, and the assemblages of oligochaetes and chironomids were analyzed. The highest values of diversity and taxonomic richness of macroinvertebrates corresponded to the Chubichaminí RW $\left(H^{\prime}=1.9 ; \mathrm{S}=\right.$ 19.25). This RW showed slightly polluted waters, being acceptable in terms of water quality. The multivariate analysis ordered the sites of Del Gato and Carnaval RWs with the highest nutrient load associated with more tolerant taxa such as the dipterans Stratiomyidae, Ephydridae, nematodes, oligochaetes and Physidae gastropods. The greatest diversity and richness of oligochaetes were recorded in the Cajaravillas and Chubichaminí RW in the warm months being the differences mainly due to Limnodrilus hoffmeisteri Claparède, 1862. The chironomid assemblage consisted of 24 taxa 
belonging to Chironominae, Orthocladiinae and Tanypodinae. The genus Chironomus Meigen was more abundant in Del Gato RW, corresponding mainly to C. calligraphus Goeldi, associated with environments with evident anthropic impact. The RWs studied showed different patterns in the proportion of functional feeding groups without a clear relation with land use. The indexes applied to the macroinvertebrate assemblage were effective in evaluating the ecological status of the analysed RWs.

Keywords: Biotic indices, benthic macroinvertebrates, chironomids, functional feeding groups, oligochaetes.

\section{INTRODUCCIÓN}

Los bañados o humedales de desborde fluvial son ambientes transicionales de perímetro indefinido, inundables, con abundante vegetación emergente conformada por comunidades serales, con escaso desarrollo de poblaciones limnéticas donde la inestabilidad es la norma evidenciando el ritmo anual por la sucesión de aspectos ligada a los cambios estacionales visibles en la cobertura vegetal. Las aguas pluviales o de rebalse quedan retenidas en depresiones poco pronunciadas del terreno permitiendo y condicionando la existencia de vegetación del tipo palustre o de suelo muy húmedo (pajonal) (Ringuelet, 1962). En los bañados la retención de sedimentos, nutrientes y otras sustancias contaminantes exógenas, suelen mejorar la calidad del hábitat permitiendo albergar en una variada vegetación acuática, una importante diversidad de meso y macrofauna, que actúan como fuente de alimento para otros grupos faunísticos superiores tales como anfibios, reptiles, aves y mamíferos (Neiff, 2001; Rodrigues Capítulo et al., 2009; Gómez et al., 2016; Armendáriz et al., 2017). Una gran diversidad de invertebrados acuáticos encuentra en estos hábitats un sitio óptimo para cumplir con el desarrollo de sus ciclos vitales e integran una parte importante de la composición biológica y de las tramas tróficas de esta zona de desborde de los ríos (Rodrigues Capítulo et al., 2004; 2010).

En las últimas decádas la limnología ha utilizado los ensambles de macroinvertebrados del bentos como una herramienta para la evaluación de la salud de los ríos (Armitage et al., 1983). Particularmente han resultado muy eficientes para reflejar contaminaciones puntuales o difusas que a veces no pueden ser detectadas por otra metodología. Las principales ventajas de utilizar a los macroinvertebrados como indicadores biológicos son que estos organismos están ampliamente distribuidos en diversos tipos de humedales $y$, cumplen todo o parte de su ciclo de vida en estos hábitats, por lo que son afectados (Barbour et al., 1999). Por otra parte, cumplen un rol fundamental en las redes tróficas (U.S. EPA, 2015). El uso de indicadores biológicos para la evaluación de las modificaciones en las condiciones del hábitat constituye una herramienta útil en la prevención de pérdida de especies (Ocon y Rodrigues Capítulo, 2004).

La complejidad de los ensambles de invertebrados puede ser estudiada desde perspectivas estructurales o funcionales. Sin embargo, muchos autores han resaltado que los estudios integrados son más apropiados para establecer los patrones de biodiversidad y para entender las variaciones de la diversidad específica (Zilli et al., 2008a). Si bien algunos índices se basan solamente en la riqueza y diversidad de especies, otros índices priorizan la presencia de determinadas especies en función de su sensibilidad a la calidad del agua. En los ambientes lóticos pampeanos se han utilizado diversos índices basados en macroinvertebrados, tales como IBPAMP (Índice Biótico Pampeano) y los scores: IMRP (Índice de Macroinvertebrados para Ríos Pampeanos), además del PBMWP (Pampean Biomonitoring Working Party) adaptado para ríos de la llanura pampeana y su score medio ASTP (Average Score per Taxon) (Rodrigues Capítulo, 1999; Rodrigues Capítulo et al., 2006, 2008, 2009).

Dentro de la comunidad bentónica los anélidos oligoquetos desempeñan un papel preponderante en los ecosistemas de aguas continentales, y a menudo, representan uno de los grupos más diverso y abundante (Wetzel et al., 2006). Su valor ecológico incluye su 
participación en los ciclos de nutrientes y en los procesos de flujo de energía (Pelegri \& Blackburn, 1995; Vorobyev et al., 2010), su impacto en la estructura de los sedimentos y su importancia en la red trófica acuática. Su larga historia como indicadores de la condición ambiental y su uso en el monitoreo de los ecosistemas acuáticos han hecho de ellos un taxón imprescindible en la práctica de biomonitoreos de ambientes dulceacuícolas (Lafont et al., 2001; Krodkiewska \& Michalik-Kucharz, 2009; Marchese, 2009; Armendáriz et al., 2011; Rodríguez \& Reynoldson, 2011). Por otro lado, la familia Chironomidae (Insecta: Diptera) es un grupo cosmopolita, cuyos estados inmaduros se encuentran en prácticamente todos los ambientes acuáticos. Generalmente representan otro de los grupos de macroinvertebrados más abundantes y con mayor riqueza taxonómica en los diferentes limnótopos (Ferrington, 2008). Se encuentran en una gran diversidad de nichos y cumplen un rol fundamental en las redes tróficas al consumir y reciclar materia orgánica, siendo un nexo entre los recursos basales y los consumidores secundarios (Pinder, 1986). Sus especies presentan diferentes tolerancias a los gradientes ambientales, así como a los impactos antropogénicos. De esta manera, los quironómidos también son utilizados ampliamente en los estudios de monitoreo, protección y conservación de ambientes acuáticos (Paggi, 1999; Cortese et al., 2019, Cortelezzi et al., 2020).

Las respuestas funcionales de los invertebrados permiten conocer y comprender diferentes procesos que tienen lugar en un ecosistema acuático, convirtiéndose en indicadores de su integridad (Bunn et al., 1999). Entre estas respuestas, las estrategias alimentarias reflejan las adaptaciones de las especies a las condiciones ambientales imperantes y su variación puede indicar cambios en dichas condiciones. En este sentido, el incremento de la influencia humana sobre los ecosistemas acuáticos, tanto en forma directa como indirecta, puede causar cambios en la alimentación de los organismos (Fenoglio et al., 2005), pudiendo aún alterar la composición de los ensambles. Establecer los grupos funcionales alimentarios para un determinado ambiente permite caracterizar el flujo de materia y energía entre dichos grupos dentro del ensamble.

El objetivo del trabajo fue caracterizar el ensamble de macroinvertebrados presentes en bañados de desborde fluvial de arroyos de llanura con diferente uso del suelo, desde un punto de vista estructural y funcional. Por otra parte, se propuso valorar la calidad ecológica de estos ambientes mediante la aplicación de índices bióticos, ecológicos y análisis multivariados. Se analizaron en particular los oligoquetos y quironómidos del complejo bentónico para observar posibles diferencias de estos taxa en los sitios previos y posteriores a los bañados en estudio.

\section{MATERIALES Y MÉTODOS Área de estudio y caracterización}

Para el presente estudio, se realizaron cuatro muestreos estacionales (otoño, invierno y primavera de 2017 y verano de 2018) en cuatro bañados del área pampeana, Buenos Aires, Argentina (Figura 1). Los bañados del $A^{\circ}$ Del Gato $\left(34^{\circ} 58^{\prime} 49.52^{\prime \prime} \mathrm{S}, 58^{\circ} 3^{\prime} 10.70^{\prime \prime} \mathrm{W}\right.$ ) y del $\mathrm{A}^{\circ}$ Carnaval (34 $55^{\circ} 5.33^{\prime \prime} \mathrm{S}, 5^{\circ} 6^{\prime} 32.24^{\prime \prime} \mathrm{W}$ ) presentan intensa actividad de horticultura y agricultura en sus cuencas mientras que los bañados del $A^{\circ}$ Cajaravillas $\left(35^{\circ} 2^{\prime} 44.52^{\prime \prime} \mathrm{S}, 57^{\circ} 48^{\prime} 43.41^{\prime \prime} \mathrm{W}\right.$ ) y el del A ${ }^{\circ}$ Chubichaminí $\left(35^{\circ} 7^{\prime} 44.50^{\prime \prime} \mathrm{S}, 57^{\circ} 41^{\prime} 51.95^{\prime \prime}\right.$ W) se encuentran en áreas con ganadería extensiva sobre pasturas naturales.

En cada muestreo se registraron in situ parámetros fisicoquímicos de rutina con sensores multiparamétricos (HORIBA): oxígeno disuelto, \% de saturación de oxígeno, conductividad, $\mathrm{pH}$, temperatura y turbidez. A partir de muestras de agua, en el laboratorio de química del ILPLA se determinaron, según la metodología propuesta por APHA (1998), los siguientes parámetros: demanda bioquímica de oxígeno (incubación de la muestra durante cinco días a $20^{\circ} \mathrm{C}$ y cálculo de la diferencia de oxígeno disuelto inicial y final), demanda química de oxígeno (método de reflujo con dicromato de potasio), nitritos (diazotación), nitratos (espectrofotometría por reducción a nitrito en una columna de cadmio), amonio (método de Azul de Indofenol), fosfatos (formación de fosfomolibdato por reducción con ácido ascórbico). La tabulación de esta información 


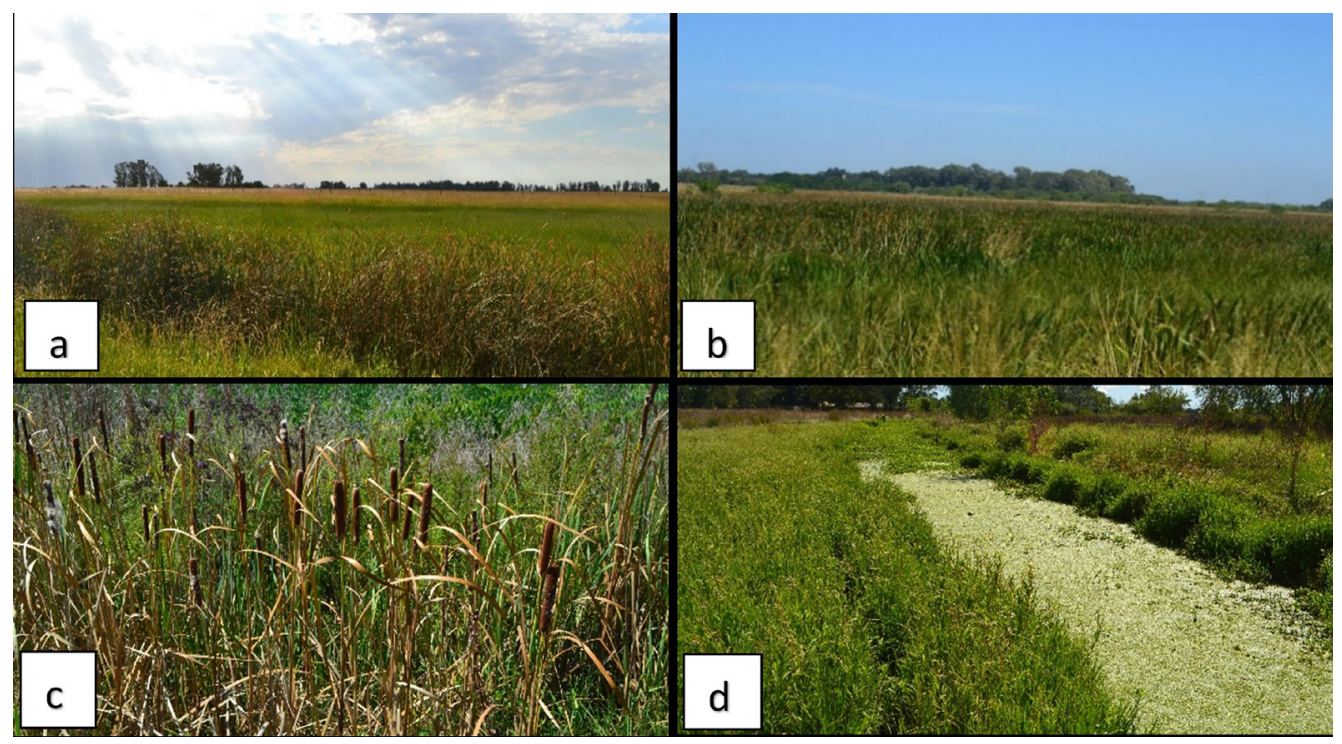

Figura 1. Bañados de la zona de estudio fotografiados durante un muestreo de verano. a: Chubichaminí; b: Cajaravillas; c: Carnaval y d: Del Gato.

Figure 1. Riverine wetlands in the study area photographed during a summer sampling. a: Chubichaminí; b: Cajaravillas; c: Carnaval y d: Del Gato.

puede observarse en los anexos del artículo de Rodrigues Capítulo et al., (2020) según las diferentes estaciones climáticas, con excepción del $A^{\circ}$ Carnaval que no pudo muestrearse en febrero de 2018 por falta de caudal de agua a causa de la intensa sequía zonal.

\section{Tareas de campo y laboratorio}

Para el estudio de los macroinvertebrados se extrajeron muestras triplicadas del sedimento con una draga Ekman $\left(100 \mathrm{~cm}^{2}\right)$ de cada sitio. El material se fijó en el campo con $5 \%$ de formaldehído. A su vez para la recolección de los quironómidos se tomaron muestras utilizando redes "D-net" en un cuadrante de $40 \times 40 \mathrm{~cm}$, las que fueron fijadas con etanol al $70 \%$.

Para el caso particular de los oligoquetos y quironómidos se consideró la extracción de muestras en los bañados propiamente dichos, pero también en los tramos del arroyo previo y posterior a los mismos, con el fin de observar posibles diferencias en la estructuración de estos dos taxa por efecto del tramo estudiado. En el laboratorio las muestras del sedimento fueron lavadas con tamices de $500 \mu \mathrm{m}$ de abertura de malla para retener los invertebrados junto al material más grueso. El material retenido fue separado bajo una lupa binocular previamente teñido con eritrosina B, para su identificación taxonómica con bibliografía especializada (Domínguez y Fernández, 2009, Lopretto y Tell, 1995 y Merritt et al., 2008), hasta el menor nivel de identificación posible para su posterior cuantificación. Particularmente para la determinación taxonómica de los oligoquetos y quironómidos se realizó un análisis más específico. Los oligoquetos se montaron en portaobjetos y clarificaron con una mezcla de ácido láctico y glicerina (50/50). Se identificaron usando un microscopio compuesto al nivel taxonómico más bajo posible de acuerdo con Brinkhurst \& Marchese (1992) y la International Commission on Zoological Nomenclature (Opinion 2167, 2007). Los ejemplares de Enchytraeidae solo fueron reconocidos como morfotipos (Schmelz \& Collado, 2010) ya que al momento no hay claves de la familia disponibles para la región. Las larvas de quironómidos previamente separadas fueron preparadas siguiendo la metodología descrita en Epler (2001). La identificación de las larvas fue 
realizada utilizando las claves de Epler (2001) y Silva et al. (2018) hasta el nivel taxonómico de género, debido a que su diferenciación a nivel de especies, en gran parte de los casos no es precisa a través del uso de esos estadios.

\section{Análisis de datos}

Para observar la estructuración de los ensambles de macroinvertebrados presentes en el complejo bentónico a partir de los datos obtenidos se estimaron la densidad, el número de taxa, la diversidad de Shannon-Wiener $\left(\mathrm{H}^{\prime}\right)$ y la equitabilidad de Pielou (J) para el ensamble en general, y para oligoquetos y quironómidos en particular. Para este último ensamble también se utilizó la distancia taxonómica propuesto por Clarke \& Warwick $(1998,1999)$.

Para analizar el ensamble de los oligoquetos se aplicó el índice de similitud de Bray-Curtis. Se realizó un test de permutación (ANOSIM) a dos vías cruzado para testear la hipótesis de diferencias en la composición de los ensambles con respecto a: a) los cuatro bañados analizados y b) los tres tramos (prebañado, bañado, postbañado) estudiados en cada caso. Para determinar la contribución de las especies a las diferencias entre los grupos analizados se aplicó la rutina SIMPER (Gómez-Lemos et al., 2010). Para el ensamble de quironómidos se realizó un Non Metric Multidimensional Scaling (NMDS). Para determinar las diferencias estadísticas entre los grupos obtenidos a partir del análisis NMDS, se realizó un test de permutación (ANOSIM) de una vía, con una permutación de 9999 y nivel de significancia de $p<0,05$.
Para estimar aquellos quironómidos que mayormente contribuyeron a las disimilitudes observadas se realizó un análisis SIMPER. Todos estos análisis se realizaron con el programa PAST (PAleontologicalSTatistics, versión 4.01, Hammer et al., 2001).

Con el propósito de determinar la calidad del agua a partir del ensamble de macroinvertebrados se aplicaron varios índices bióticos tales como el IBPAMP (Índice Biótico Pampeano) (Rodrigues Capítulo et al., 2001), el PBMWP (Pampean Biomonitoring Working Party) y su escore ASPT (Average Score Per Taxon) (Rodrigues Capítulo et al., 2006, 2008) derivados del IBMWP (para ríos Ibéricos de Alba Tercedor, et al. (2002) con el agregado de familias de macroinvertebrados de la Ecorregión Pampa. Para el ordenamiento de los datos de los bañados según los diversos índices bióticos utilizados para los ensambles de macroinvertebrados se adjunta una tabla de referencia (Tabla 1), donde se detallan los valores entre los que fluctúan cada uno y sus respectivas clases de calidad ecológica.

Con el fin de analizar la relación entre las variables ambientales y el ensamble de macroinvertebrados para los diferentes sitios de estudio, se utilizaron análisis multivariados. Para observar si las variables en su conjunto respondían a un modelo lineal (RDA: Redundance Analysis) o unimodal (CCA Canonical Correspondence Analysis) se realizó un DCCA (Detrended Correspondence Canonical Analysis) utilizando el Programa CANOCO (Windows) (ter Braak (1986); ter Braak \& Verdonschot (1995) con el fin de determinar la longitud del gradiente y optar por algunos de los métodos antes mencionados (Zornoza, 2015).

Tabla 1. Valores de los índices bióticos y su significado de calidad de aguas.

Table 1. Values of biotic indices and their meaning of water quality.

\begin{tabular}{clcccl}
\hline Clases & Calidad & IBPAMP & PBMWP & ASPT & Significado \\
\hline I & Buena & $>14$ & $>125$ & $>4,3$ & Aguas muy limpias \\
II & Aceptable & $8-9$ & $91-110$ & $3,0-3,7$ & $\begin{array}{l}\text { Aguas donde se evidencian algunos } \\
\text { efectos de la contaminación }\end{array}$ \\
III & Dudosa & $6-7$ & $51-70$ & $2,6-2,9$ & $\begin{array}{l}\text { Aguas moderadamente } \\
\text { contaminadas }\end{array}$ \\
IV & Critica & $4-5$ & $15-50$ & $2,1-2,5$ & Aguas muy contaminadas \\
V & Muy crítica & $1-3$ & $<15$ & $1-2,0$ & Aguas fuertemente contaminadas \\
\hline
\end{tabular}


Luego se realizó un análisis ponderado de los taxa con el propósito de evaluar las relaciones entre los conjuntos de invertebrados bentónicos y las variables ambientales. El ajuste de los análisis mencionados fue realizado mediante la prueba de Monte Carlo considerando la significancia del primer eje y el total de éstos. Los macroinvertebrados hallados en los diferentes sitios fueron asignados a su correspondiente grupo funcional alimentario (GFA) de acuerdo con las categorías definidas por Cummins (1973), Merritt \& Cummins (1996), Cummins et al. (2005), Merritt et al. (2008), Ramírez \& Gutiérrez Fonseca (2014), Zilli et al. (2008b) y a observaciones previas de los autores (Tabla 2). A partir de la densidad de los organismos presentes se obtuvo la proporción de grupos funcionales alimentarios para cada arroyo.

Tabla 2. Macroinvertebrados registrados en los arroyos estudiados: Chubichaminí (CH), Cajaravillas (CJ), Carnaval (CR) y Del Gato (GA). Las abreviaturas (Abr.) de los taxa son las usadas en la Figura 6. En negrita se indican algunos taxa que fueron utilizados para aplicar índices bióticos. GFA: grupos funcionales alimentarios más comunes asignados a cada taxón. D: depredador; C-R: colector-recolector; C-F: colector-filtrador; R: raspador y F: fragmentador.

Table 2. Macroinvertebrates recorded in the streams studied: Chubichaminí (CH), Cajaravillas (CJ), Carnaval (CR) and Del Gato (GA). The abbreviations (Abr.) of the taxa are those used in Figure 6. Some taxa that were used to apply biotic indices are indicated in bold. GFA: the most common food functional groups assigned to each taxon are indicated. D: predator; C-R: gathering-collector; C-F: collector-filter; R: scraper and F: shredders.

\begin{tabular}{|c|c|c|c|c|c|c|c|c|c|c|c|c|c|}
\hline Taxa & Abr. & GFA & $\mathrm{CH}$ & CJ & CR & GA & Taxa & Abr. & GFA & $\mathrm{CH}$ & CJ & CR & GA \\
\hline Hydridae & Hydr & D & $x$ & & & & Palaemon & & $C-R$ & $\mathbf{x}$ & & & \\
\hline TURBELARIA & Turbel & D & $x$ & & $\mathrm{x}$ & $x$ & Isotomidae & Isotomi & $C-R$ & $x$ & $x$ & $x$ & $x$ \\
\hline Girardia & Girardia & D & $x$ & $x$ & & & Entomobridae & Entom & $C-R$ & & & & $x$ \\
\hline Temnocephala & Temno & D & & $x$ & & & Coenagrionidae & Coenagri & $\mathrm{D}$ & $x$ & $x$ & $x$ & $x$ \\
\hline NEMERTEA & Nemertea & D & $x$ & & $x$ & & Libellulidae & Libellul & D & & $x$ & & \\
\hline NEMATODA & Nemat & - & $x$ & $x$ & $x$ & $x$ & Gomphidae & Gomph & D & & & & \\
\hline OLIGOCHAETA & Olig & $C-R$ & $x$ & $x$ & $x$ & $x$ & Caenis & Caenis & $C-R$ & $x$ & $x$ & $x$ & \\
\hline HIRUDINEA & Hirud & D & $x$ & $x$ & $x$ & $x$ & Americabaetis & America & $C-R$ & $x$ & $x$ & $x$ & \\
\hline Eupera & Eupera & $C-F$ & $x$ & $x$ & $x$ & & Campsurus & Campsur & $\mathrm{C}-\mathrm{F}$ & $x$ & & & \\
\hline Pisidium & Pisidium & $C-F$ & $x$ & $x$ & $x$ & & Chironomidae & Chiron & $C-R$ & $x$ & $x$ & $x$ & $x$ \\
\hline Musculium & Muscul & $C-F$ & $x$ & & & & Ceratopogonidae & Ceratop & $C-R$ & $x$ & $x$ & $x$ & \\
\hline Diplodon & Dipl & $C-F$ & $x$ & & & & Stratiomyidae & Stratiom & $C-R$ & & $x$ & & $x$ \\
\hline Biomphalaria & Biomph & $\mathrm{R}$ & & $x$ & & & Sciomyzidae & & $\mathrm{D}$ & & & $\mathbf{x}$ & \\
\hline Physa & Physa & $\mathrm{R}$ & & & $x$ & $x$ & Ephydridae & Ephyd & $C-R$ & $x$ & & $x$ & \\
\hline Uncancylus & Uncancy & $\mathrm{R}$ & $x$ & $x$ & $x$ & $x$ & Muscidae & & $C-R$ & & & $\mathbf{x}$ & \\
\hline Hebetangylus & Hebet & $\mathrm{R}$ & & $x$ & & & Simulidae & Simulida & $\mathrm{C}-\mathrm{F}$ & $x$ & & & \\
\hline Pomacea & Pomacea & $\mathrm{R}$ & $x$ & & $x$ & $x$ & Hydrophilidae & Hydrophi & $C-R$ & $x$ & $x$ & & \\
\hline Drepanotrema & Drep & $\mathrm{R}$ & $x$ & $x$ & $x$ & $x$ & Berosus & Berosus & $C-R$ & $x$ & $x$ & & \\
\hline Heleobia & Hele & $\mathrm{R}$ & $x$ & $x$ & $x$ & $x$ & Elmidae & Elmidae & $\mathrm{R}$ & $x$ & & & \\
\hline CYCLOPOIDA & Cycl & D & $x$ & $x$ & $x$ & $x$ & Dytiscidae & Dytis & D & $x$ & & & $x$ \\
\hline CLADOCERA & Clad & $C-F$ & $x$ & $x$ & & & Belostomatidae & & D & & & & $\mathbf{x}$ \\
\hline Argulus & Argul & D & $x$ & & & & Corixidae & Corixida & $\mathrm{D}$ & $x$ & $x$ & $x$ & \\
\hline OSTRACODA & Ostr & $\mathrm{C}-\mathrm{F}$ & $x$ & $x$ & & $x$ & Hydroptila & Hydropti & $C-R$ & $x$ & & & \\
\hline Hyalella & Hyalella & $\mathrm{F}$ & $x$ & $x$ & & $x$ & HYDRACHNIDIA & Hydrac & D & $x$ & $x$ & $\mathrm{x}$ & $x$ \\
\hline Silviocarcinus & Silvioca & $C-R$ & $x$ & & & & & & & & & & \\
\hline
\end{tabular}




\section{RESULTADOS}

\section{Ensamble de macroinvertebrados}

El registro de los taxa de macroinvertebrados (con diferentes niveles de identificación taxonómica) entre los cuatro bañados de desborde fluvial, y considerando cuatro muestreos realizados, se dispuso en la Tabla 2.

La densidad media de los macroinvertebrados (y microcrustáceos retenidos en tamices de 500 $\mu \mathrm{m})$ fue en general diferente para los cuatro bañados estudiados. Las mayores densidades medias correspondieron a los bañados del $A^{\circ}$ Carnaval y Chubichaminí que superaron los 40000 ind. $/ \mathrm{m}^{2}$, en tanto que el bañado del $A^{\circ}$ Cajaravillas albergó alrededor de 16000 ind./ $\mathrm{m}^{2} \mathrm{y}$ el del $\mathrm{A}^{\circ}$ Del Gato con algo menos de 6000 ind./ $\mathrm{m}^{2}$. En todos los casos los taxa dominantes numéricamente fueron los oligoquetos seguidos por los bivalvos y dípteros en el caso del Chubichaminí, por los macrocrustáceos en el Cajaravillas, por los bivalvos y gasterópodos en el Carnaval y por los microcrustáceos en el Del Gato (Figura 2).

Los mayores valores estimados de diversidad $\left(\mathrm{H}^{\prime}\right)$ y riqueza $(\mathrm{S})$ taxonómica correspondieron al bañado Chubichaminí $\left(H^{\prime}=1,9 ; S=19,25\right)$, seguido por los del Cajaravillas $\left(H^{\prime}=1,86\right.$; $S=17,5)$, el Carnaval $\left(H^{\prime}=1,76 S=18,7\right)$, y por último los del bañado Del Gato $\left(H^{\prime}=1,54 ; S=\right.$ $11,3)$. En cuanto a la equitatividad los cuatro bañados presentaron valores similares $(V=0,63)$, (Figura 3).

Los resultados promedio estimados para los índices bióticos reflejaron mayores diferencias entre los bañados en el mismo orden señalado para los valores de diversidad. El bañado del $A^{\circ}$ Chubichaminí manifestó un estado aceptable y con leves efectos de contaminación en cuanto a la calidad del agua en base al ensamble de los macroinvertebrados (sin considerar la mayoría de aquellos taxa presentes en macrófitas) reflejado por los índices PBMWP, IBPAMP y ASPT (Figura 4). Los desvíos estándar presentan una fluctuación que seguramente indica diferencias estacionales entre los muestreos. Con relación a los bañados de los arroyos Cajaravillas y Carnaval los valores de los índices son aproximadamente los mismos reflejando una característica moderadamente poluída o
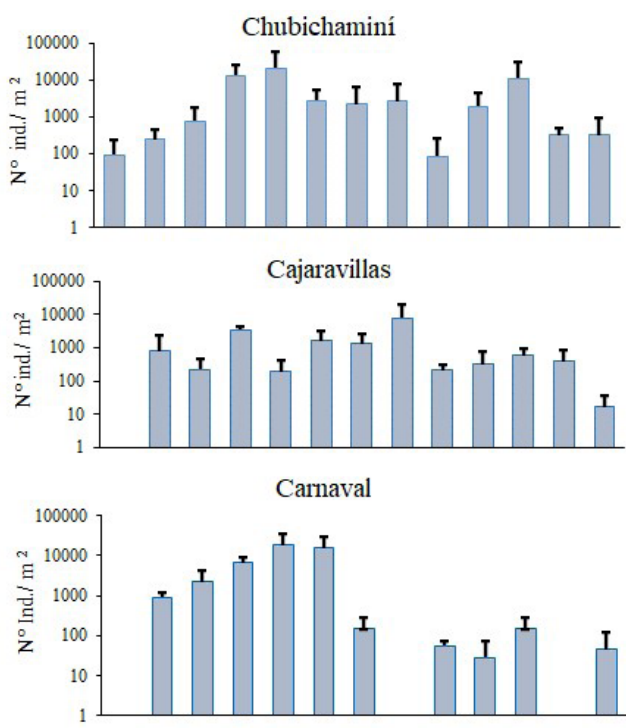

Del Gato

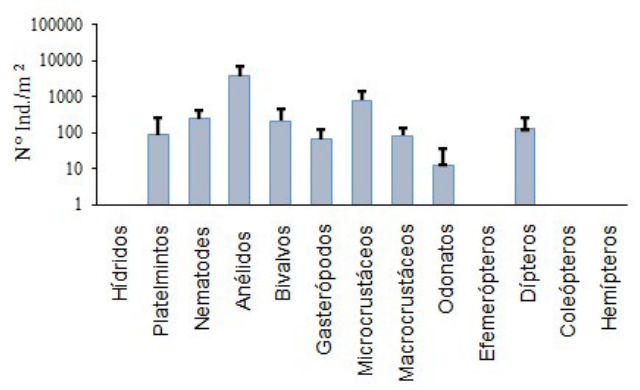

Figura 2. Densidad promedio (ind./ $/ \mathrm{m}^{2}+\mathrm{DS}$ ) en escala logarítmica de los taxa dominantes de invertebrados bentónicos de los bañados de desborde fluvial en las cuatro estaciones de muestreo.

Figure 2. Average density (ind. $/ \mathrm{m}^{2}+\mathrm{SD}$ ) on a logarithmic scale of the dominant taxa of benthic invertebrates in the riverine wetlands in the four sampling stations.

contaminada. En cambio, en el bañado del Del Gato los tres índices indican aguas fuertemente poluídas o muy contaminadas (Figura 4).

\section{Taxa y variables ambientales}

Debido a que el valor obtenido de la longitud de gradientes del análisis del DCCA fue 3,25 (Tabla 2) se utilizó un análisis CCA para el tratamiento del conjunto de datos correspondientes a las variables ambientales, sitios y macroinvertebrados. Las variables elegidas, luego de descartar aquellas que tenían alta colinealidad y valores de inflación 


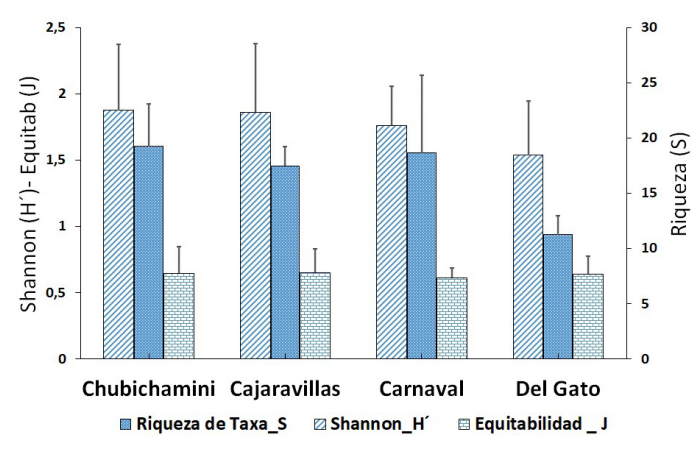

Figura 3. Promedio (+DS) de los Índices de diversidad de Shannon -Wiener $\left(\mathrm{H}^{\prime}\right)$, riqueza taxonómica (S) y equitabilidad (J).

Figure 3. Average and (+SD) of the Shannon -Wiener diversity indices $\left(\mathrm{H}^{\prime}\right)$, taxonomic richness $(\mathrm{S})$ and equitability (J).

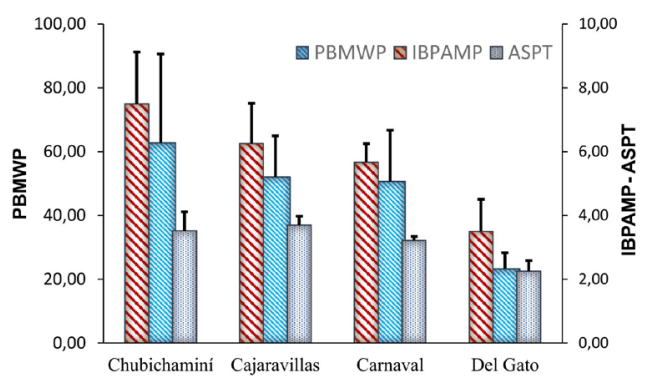

Figura 4. Valores medios (+ DS) de los índices bióticos PBMWP, IBPAMP y su escore medio ASPT en los cuatro bañados analizados.

Figure 4. Mean values (+ SD) of the biotic indices PBMWP, IBPAMP and their mean ASPT score in the four analyzed riverine wetlands.

superiores a 20 fueron: temperatura $\left({ }^{\circ} \mathrm{C}\right)$, conductividad (cond), \% de saturación de oxígeno disuelto (\% OD), turbidez (turb), total de sólidos en suspensión (TDS), fosfatos ( $\left(\mathrm{PO}_{4}\right)$, nitratos $\left(\mathrm{N}-\mathrm{NO}_{3}\right)$, nitritos $\left(\mathrm{N}-\mathrm{NO}_{2}\right)$, nitrógeno total (Ntotal) y amonio $\left(\mathrm{N}-\mathrm{NH}_{4}\right)$.

El porcentaje explicado de la varianza para la relación especies- variables ambientales para el Eje I fue de $28 \%$ y para el eje II $24,7 \%$ alcanzando el $84,5 \%$ en los cuatro primeros ejes con alta significación estadística de acuerdo con la prueba de Montecarlo (0,016 para el primer eje, y 0,002 para el conjunto de los ejes canónicos, Tabla 3). Los dos primeros ejes del CCA fueron representados en un gráfico de ordenamiento de las variables ambientales y los sitios de muestreo (Figura 5) y el ordenamiento de los taxa respecto de los sitios puede verse en la Figura 6.

En el cuadrante conformado por el lado positivo del eje I y negativo del eje II, donde adquirieron importancia las variables TSD, $\mathrm{N}-\mathrm{NH}_{4}, \mathrm{P} \mathrm{PO}_{4}$ y N Total, se ubicaron la mayoría de los sitios correspondientes a los bañados de los arroyos Del Gato y Carnaval, si bien también se ordenaron hacia el extremo derecho del eje I con mayor influencia de los nitritos las estaciones muestreadas en verano del $A^{\circ}$ Chubichaminí. En este sector predominaron los taxa de organismos más tolerantes a la perturbación como los dípteros Stratiomyidae y Ephydridae, los nematodes, los oligoquetos, los gasterópodos Physidae, Hydrobiidae (Heleobia sp.), Drepanotrema sp., los Ancylidae, Ios coleópteros Dytiscidae y los ácaros Hydrachnida. En el cuadrante negativo de los dos ejes, donde la principal variable fue la turbidez, además de las variables que actúan sobre el eje I en su parte negativa $\left(T^{\circ} \mathrm{C}\right.$ y $\left.\% O D\right)$ se ubicó el bañado del $A^{\circ}$ Chubichaminí de primavera donde predominaron los Coleópteros Elmidae, los efemerópteros Americabaetis sp. y Campsurus sp., los tricóperos Hydroptilidae, los bivalvos Pisidium sp. y Musculium sp. y también los Chironomidae. En invierno este sitio se dispuso sobre el eje Il y se ubicaron los bivalvos Eupera sp. y Diplodon delodontus (Lamarck, 1819) y el

Tabla 3. Resumen de los valores obtenidos para el Análisis DCCA y CCA de los bañados en estudio. LC: longitud del gradiente. IT: inercia total. Corr. sp. var. amb.: correlación entre especies y variables ambientales. \%ac. var. rel. sp. var. amb.: porcentaje acumulado de la varianza de la relación entre especies y variables ambientales.

Table 3. Summary of the values obtained for the DCCA and CCA analysis of the studied riverine wetlands. LC: gradient length. IT: total inertia. Corr. sp. var. amb.: correlation between species and environmental variables. \%ac. var. rel. sp. var. amb.: cumulative percentage of the variance of the relationship between species and environmental variables.

\begin{tabular}{lccccc}
\hline Ejes & I & II & III & IV & IT \\
LC (DCCA) & 3,255 & 2,117 & 1,479 & 1,609 & \\
Resumen CCA & & & & & \\
Autovalores & 0,631 & 0,555 & 0,407 & 0,304 & 2,49 \\
Corr. sp var. amb. & 0,989 & 0,984 & 0,975 & 0,972 & \\
\%ac. var. rel. sp. var. amb. & 28,1 & 52,8 & 70,9 & 84,5 &
\end{tabular}

Test Montecarlo 1 eje canónico, P-valor=0,016 y total de ejes P-valor $=0$ 0,002 


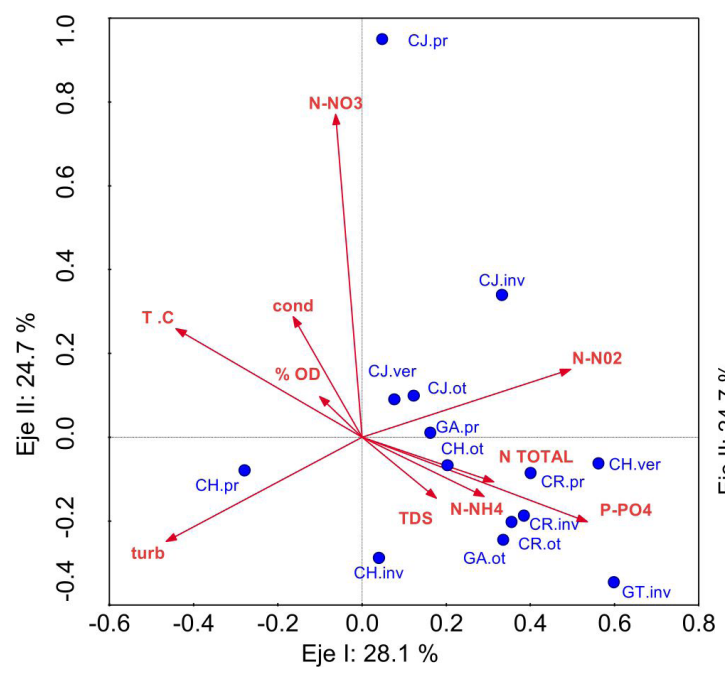

Figura 5. Ordenamiento de sitios y variables ambientales en los ejes canónicos (CCA) relacionados con los ensambles de macroinvertebrados de los bañados de desborde pertenecientes a cuencas del Gran La Plata y Magdalena (Buenos Aires). CH: Chubichaminí; CJ: Cararavillas; CR: Carnaval; GA: Del Gato; pr: primavera; ver; verano; ot: otoño; inv: invierno.

Figure 5. Ordering of sites and environmental variables in the canonical axes (CCA) related to the macroinvertebrate assemblages of the RWs belonging to the Greater La Plata and Magdalena basins (Buenos Aires). CH: Chubichaminí; CJ: Cararavillas; CR: Carnaval; GA: Del Gato; pr: spring; ver: summer; ot: autumn; inv: winter.

cangrejo Sylviocarcinus sp. Sobre el cuadrante formado entre el lado positivo de ambos ejes donde se disponen las variables de nitrógeno $\left(\mathrm{N}-\mathrm{NO}_{2} \text { y N-NO}\right)_{3}$ ) se ubicaron todas las muestras correspondientes al bañado del $\mathrm{A}^{\circ}$ Cajaravillas. Los principales macroinvertebrados asociados a este sector fueron los anfípodos Hyalellidae, temnocéfalos, los odonatos Coenagrionidae, Gomphidae y Libellulidae, y los coleópteros Hydrophilidae entre los principales.

\section{Ensambles de oligoquetos y quironómidos}

El ensamble de los anélidos (no Hirudinea) estuvo conformado por 38 taxa pertenecientes a cuatro familias: Naididae (32 especies), Opistocystidae (una especie), Enchytraeidae (cuatro morfotipos) y Aeolosomatidae (una especie). En el período estudiado los mayores valores de riqueza y diversidad se registraron

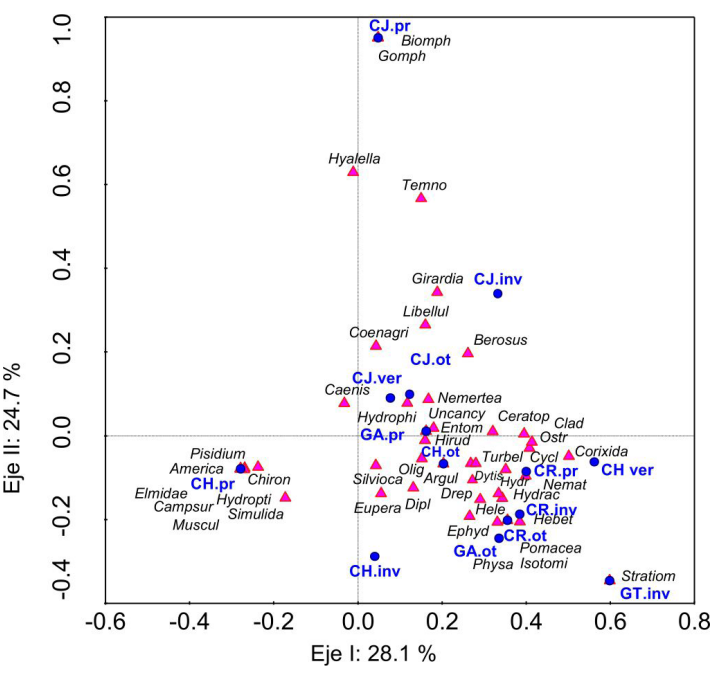

Figura 6. Ordenamiento de sitios y taxa del ensamble de invertebrados en los ejes canónicos (CCA) de cuatro bañados de desborde pertenecientes a cuencas del gran La Plata y Magdalena (Buenos Aires). Las abreviaturas utilizadas en los gráficos pueden observarse en la Tabla 2 y en la leyenda de la Figura 5. Figure 6 . Ordering of sites and taxa of the invertebrate assemblage in the canonical axes (CCA) of four RWs belonging to basins of the Gran La Plata and Magdalena (Buenos Aires). The abbreviations used in the graphs can be seen in Table 2 and the legend of Figure 5.

en los arroyos Cajaravillas y Chubichaminí (Figura 7), especialmente en los meses más cálidos del año, mientras que los tres tramos analizados (prebañado, bañado y posbañado) no presentaron un patrón definido respecto a estos índices. El análisis de similitud ANOSIM indicó que había diferencias en los ensambles de los distintos bañados (R global: 0,218, p: $0,0005)$ y también entre los tramos estudiados ( $R$ global: 0,186, p: 0,0073), sin embargo no podrían ser interpretados como independientes, puesto que el R global fue menor a 0,75. De todos modos puede observarse un predominio de representantes de la familia Naididae en los cuatro arroyos. En el bañado del $\mathrm{A}^{\circ}$ Carnaval se observó un incremento de los Aeolosomatidae en septiembre que llevó a una preponderancia de este taxón sobre las demás familias en la siguiente estación climática. Los Enchytraeidae se registraron principalmente en los meses fríos, mientras que los Opistocystidae se presentaron 

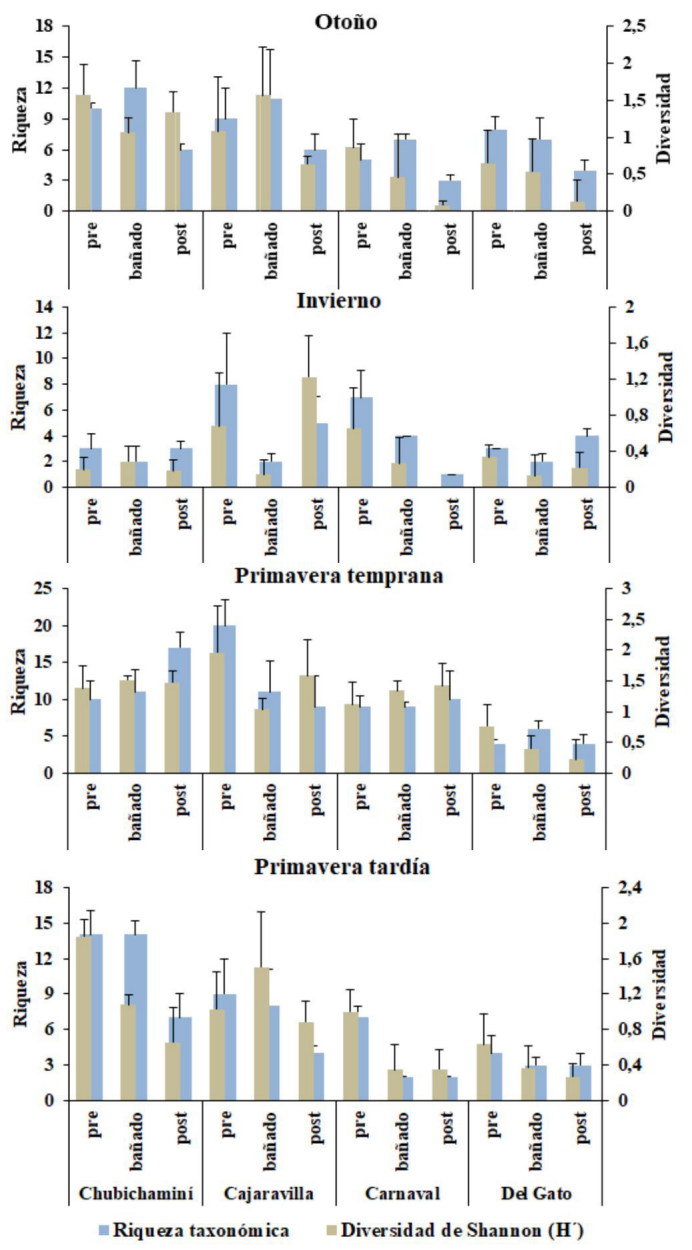

Figura 7. Riqueza específica y diversidad de ShannonWiener del ensamble de oligoquetos bentónicos en los sitios estudiados de los arroyos analizados.

Figure 7. Specific richness and Shannon-Wiener diversity of the benthic oligochaete assemblage in the studied sections of the analyzed streams.

en los meses cálidos, pero sólo en el bañado del $\mathrm{A}^{\circ}$ Cajaravillas.

En la Tabla 4 se exponen los valores del análisis SIMPER donde se indican los taxa de oligoquetos que más contribuyeron a la disimilitud entre los arroyos estudiados (disimilitud promedio: 61,33). Los tramos analizados (prebañado, bañado y posbañado) presentaron diferencias entre sí (disimilitud promedio: 68,73) principalmente debido a los taxa listados en la Tabla 5.

En el ensamble de quironómidos se colectaron 24 taxones pertenecientes a las subfamilias Chironominae $(n=10)$, Orthocladiinae $(n=8)$ y Tanypodinae $(n=6)$. Los géneros
Tabla 4. Análisis SIMPER del ensamble de oligoquetos. Taxa que contribuyeron en mayor medida a la disimilitud entre los bañados de los arroyos analizados. DP: disimilitud promedio; \% Con.: porcentaje de contribución; \% Ac.: porcentaje acumulado.

Table 4. SIMPER analysis of the oligochaete assemblage. Taxa that most contributed to the dissimilarity between the analyzed RWs.

\begin{tabular}{lccc}
\hline Taxon & DS & $\%$ Con. & $\%$ Ac. \\
\hline Limnodrilus hoffmeisteri & 37,85 & 61,72 & 61,72 \\
Aeolosoma sp & 6,98 & 11,39 & 73,11 \\
Tubifex tubifex & 6,08 & 9,92 & 83,03 \\
Bothrioneurum americanum Beddard, & & & \\
1894 & 2,16 & 3,52 & 86,55 \\
Enchytraeidae sp 1 & 1,91 & 3,12 & 89,67 \\
Limnodrilus neotropicus Cernosvitov, & & & \\
1939 & 1,88 & 3,069 & 92,74 \\
Nais pardalis Piguet, 1906 & 1,18 & 1,925 & 94,66 \\
Dero digitata (Müller, 1773) & 0,67 & 1,086 & 95,75 \\
\hline
\end{tabular}

Tabla 5. Análisis SIMPER del ensamble de oligoquetos. Taxa que contribuyeron en mayor medida a la disimilitud entre los tramos (prebañado, bañado y postbañado) analizados. DP: disimilitud promedio; \% Con.: porcentaje de contribución; \% Ac.: porcentaje acumulado.

Table 5. SIMPER analysis of the oligochaete assemblage. Taxa that most contributed to the dissimilarity between the sections analyzed.

\begin{tabular}{lccc}
\hline Taxon & DP & \% Con. & $\%$ Ac. \\
\hline Limnodrilus hoffmeisteri & 35,74 & 52,01 & 52,01 \\
$\begin{array}{l}\text { Dero obtusa d'Udekem } \\
1885\end{array}$ & 5,94 & 8,64 & 60,65 \\
Aulodrilus pigueti & 4,59 & 6,67 & 67,32 \\
Tubifex tubifex & 4,57 & 6,65 & 73,97 \\
Bothrioneurum & & & \\
americanum & 2,35 & 3,42 & 77,39 \\
Aeolosoma sp & 2,29 & 3,33 & 80,72 \\
Dero digitata & 2,02 & 2,94 & 83,65 \\
Enchytraeidae sp 1 & 1,91 & 2,78 & 86,44 \\
\hline
\end{tabular}

Chironomus Meigen, Goeldichironomus Fittkau, Parachironomus Lenz, Polypedilum Kieffer, Rheotanytarsus Thienemann et Bause, Tanytarsus van der Wulp, Coelotanypus Kieffer, Labrundinia Fittkau y Thienemanniella Kieffer estuvieron presentes en todos los ambientes, aunque para algunos géneros sus proporciones han variado en los diferentes arroyos. El género Chironomus fue más abundante en el arroyo Del Gato. A partir de las larvas de 4to estadio de Chironomus colectados en este arroyo, se pudo corroborar que la mayor parte corresponden a C. calligraphus Goeldi (Figura 8). El análisis de los datos por sitio de muestreo aplicando NMDS dio como resultado que no existe agrupamiento 
Dado que a partir de este análisis se observa que en cada estación del año los arroyos Del Gato y Carnaval se agrupan y en menor medida los arroyos Chubichaminí y Cajaravillas, se procedió a realizar un ANOSIM de una vía, con una permutación de 9999, para determinar las

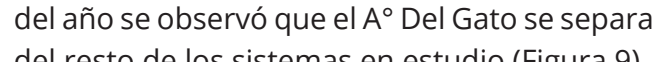

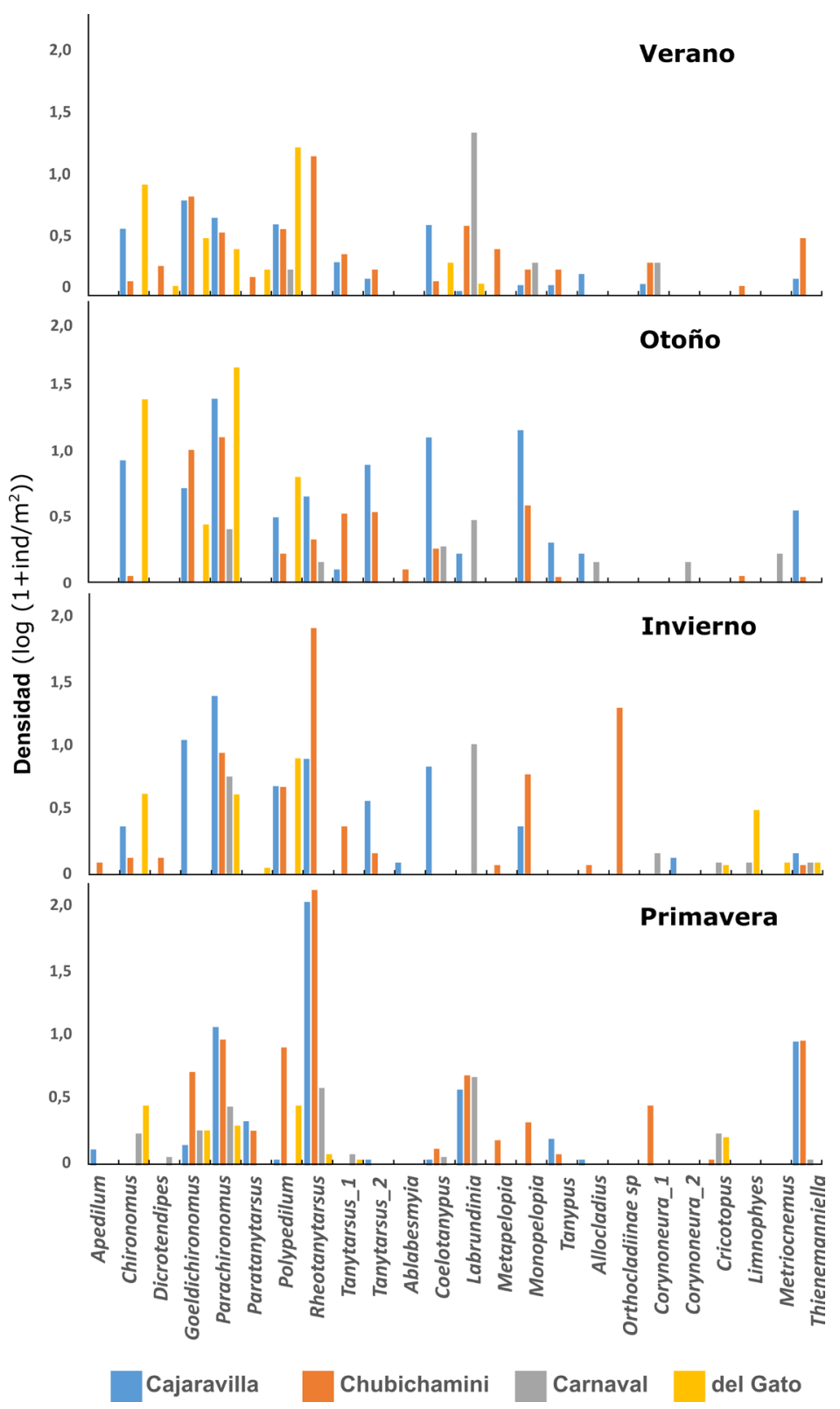

Figura 8. Densidad (escala logarítmica) del $\mathrm{N}^{\circ}$ ind./ $\mathrm{m}^{2}$ de los géneros de Chironomidae según los bañados de los diferentes arroyos estudiados.

Figure 8. Density (logarithmic scale) of the $\mathrm{N}^{\circ}$ ind. $/ \mathrm{m}^{2}$ of the genera of Chironomidae according to the RWs of the different streams studied. 


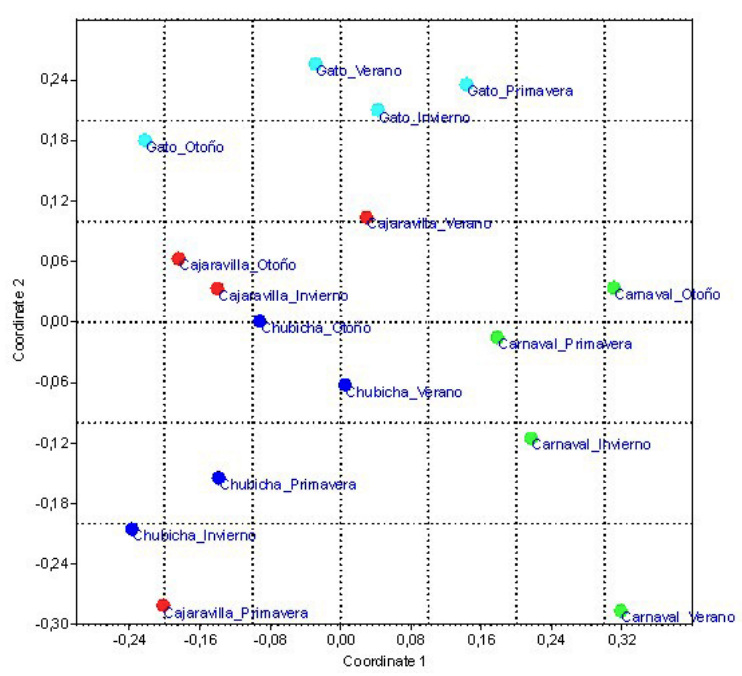

Figura 9. Análisis con NMDS de los quironómidos unificando los datos por arroyo y estación del año. Figure 9. NMDS analysis of chironomids unifying data by stream and season of the year.

diferencias estadísticas entre los grupos con un nivel de significancia $p<0,05$ entre los arroyos. Como resultado se puede observar en la Tabla 7 que no existen diferencias significativas entre los arroyos (valores de $p>0,05$ ), si bien los valores de $\mathrm{R}$ entre los mismos son cercanos a 1 excepto para los bañados de los arroyos Chubichamini y Cajaravillas, Del Gato y Cajaravillas. Para estimar aquellos taxa que mayormente contribuyeron a las disimilitudes observadas se realizó un análisis SIMPER (Tabla 8). El taxón que más contribuyó a la disimilitud fue Rheotanytarsus, cuyas larvas a menudo se asocian con comunidades de plantas acuáticas, donde construyen tubos en hojas y tallos. En aquellos pares de arroyos que presentaron valores de $\mathrm{R}>0,7$ se realizó un análisis SIMPER con el fin de determinar los taxa que más contribuyeron a dicha disimilitud. En general se mantuvieron los mismos resultados que los obtenidos en el análisis total, excepto en el par $A^{\circ}$ Carnaval- $A^{\circ}$ Del Gato donde el taxón más importante fue Labrundinia.

La riqueza (S) anual fue más alta en los arroyos con menor grado de perturbación (Tabla 6; Figura 10). A pesar de ello, los valores de diversidad $\left(\mathrm{H}^{\prime}\right)$ no muestran una disminución en aquellos arroyos con mayor perturbación e inclusive los índices de equitabilidad y dominancia son congruentes entre sí, donde expresan un ensamble con pocos individuos por especie, sin ninguna especie dominando. En donde existen diferencias entre los arroyos de menor perturbación contra los más afectados, es en la densidad de individuos y en la composición de especies (Figura 8). El índice de distancia taxonómica presentó su valor más bajo en el bañado del A $^{\circ}$ Del Gato (Tabla 6). Por otro lado, se observaron mayores valores de riqueza taxonómica y de diversidad $\left(\mathrm{H}^{\prime}\right)$ en los arroyos Cajaravillas y Chubichaminí en verano y otoño, mientras que en invierno y primavera los valores fueron similares en todos los arroyos (Tabla 6; Figura 10).

Tabla 6. Densidad e índices de diversidad, dominancia, equitabilidad y distancia taxonómica de las larvas de ensamble de Chironomidae. Chubichaminí $(\mathrm{CH})$, Cajaravillas (CJ), Carnaval (CR) y Del Gato (GA).

Table 6. Density and indices of diversity, dominance, equitability and taxonomic distance of the assemblage larvae of Chironomidae. Chubichamini $(\mathrm{CH})$, Cajaravillas (CJ), Carnaval (CR), y Del Gato (GA).

\begin{tabular}{lcccc}
\hline & CH & CJ & CR & GA \\
\hline Taxa (S) & 22 & 18 & 17 & 14 \\
Densidad (Ind./m²) & 432 & 444 & 85 & 185 \\
Dominancia (D) & 0,33 & 0,24 & 0,43 & 0,29 \\
Diversidad (H') & 1,74 & 1,86 & 1,43 & 1,43 \\
Evenness $\mathrm{e}^{\wedge} \mathrm{H} / \mathrm{S}$ & 0,26 & 0,36 & 0,25 & 0,30 \\
Equitabilidad (J) & 0,56 & 0,64 & 0,50 & 0,54 \\
Distancia taxonómica & 2,79 & 2,72 & 2,89 & 1,19 \\
\hline
\end{tabular}

\section{Relaciones tróficas}

De los 49 taxa hallados, uno de ellos correspondió al GFA fragmentador, del resto ocho correspondieron a colectores-filtradores, ocho a raspadores, 16 a depredadores y 15 a colectores-recolectores (Tabla 2). No se incluyeron en el análisis los nematodes por ser un grupo con alta variabilidad de GFA.

Los cuatro bañados estudiados mostraron diferentes patrones en la proporción de GFA. El bañado Del Gato presentó una mayor proporción de macroinvertebrados pertenecientes al grupo colector-recolector (65\%) seguido por una proporción alta de depredadores (25\%) a diferencia del Carnaval que se caracterizó por una proporción alta de colectores-filtradores (42\%) y de raspadores (37\%). Por otro lado, 

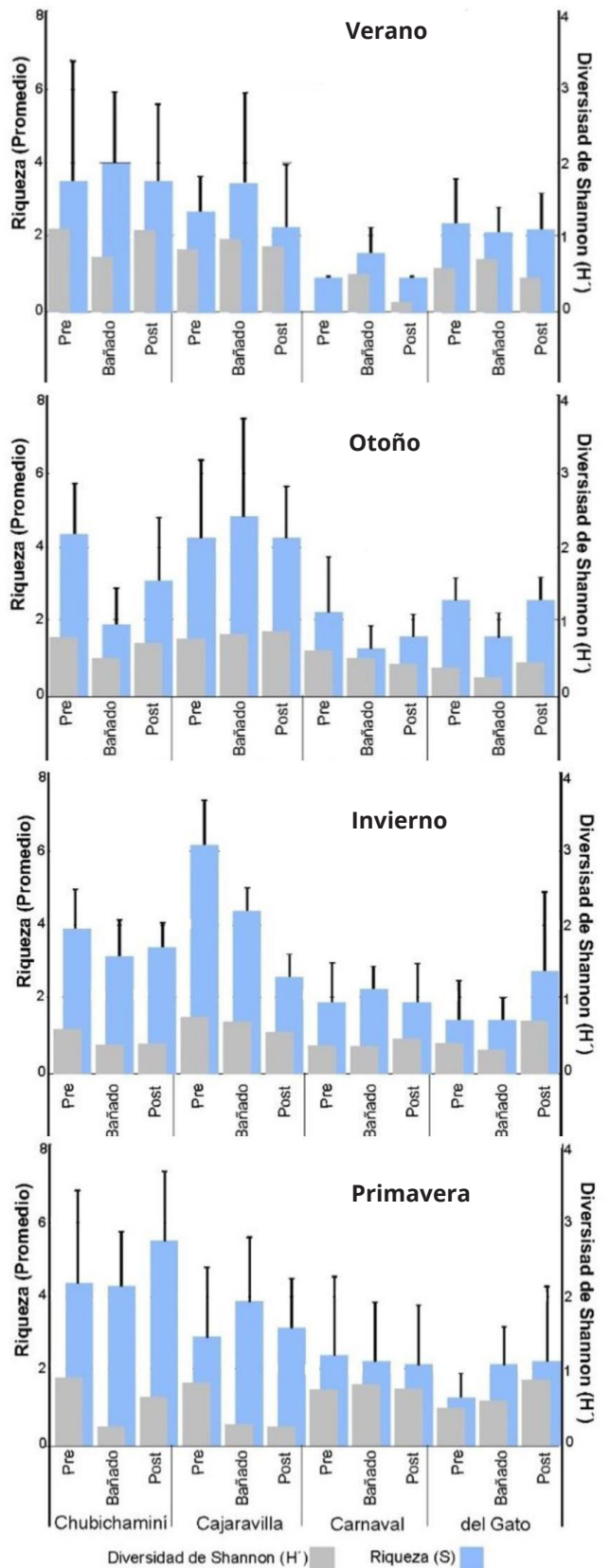

Figura 10. Riqueza taxonómica (S) e índices de diversidad $\mathrm{H}^{\prime}$ de Chironomidae.

Figure 10. Taxonomic richness $(\mathrm{S})$ and $\mathrm{H}^{\prime}$ diversity indices of Chironomidae.

el bañado Cajaravillas presentó una alta proporción de fragmentadores (46\%) debido a la alta densidad de Hyalella Smith, 1874 y por último el del Chubichaminí se caracterizó por la presencia de colectores-filtradores (56\%) y de colectores-recolectores (35\%).
Tabla 7. Densidad e índices de diversidad, dominancia, equitabilidad y distancia taxonómica de las larvas de ensamble de Chironomidae. Chubichaminí $(\mathrm{CH})$, Cajaravillas (CJ), Carnaval (CR) y Del Gato (GA).

Table 7. Densidad e índices de diversidad, dominancia, equitabilidad y distancia taxonómica de las larvas de ensamble de Chironomidae. Chubichaminí $(\mathrm{CH})$, Cajaravillas (CJ), Carnaval (CR) y Del Gato (GA).

\begin{tabular}{lcccc}
\hline & $\mathrm{CH}$ & $\mathrm{CJ}$ & $\mathrm{CR}$ & $\mathrm{GA}$ \\
\hline $\mathrm{CH}$ & 0 & & & \\
$\mathrm{CJ}$ & $-0,1042$ & 0 & & \\
$\mathrm{CR}$ & 0,7083 & 0,7917 & 0 & \\
$\mathrm{GA}$ & 0,2604 & 0,8333 & 0,7917 & 0 \\
\hline
\end{tabular}

\section{DISCUSIÓN Y CONCLUSIONES}

Según los trabajos e informes disponibles (Millennium Ecosystem Assessment, 2005; Davidson, 2014; Rodrigues Capítulo et al., 2010), la degradación de los humedales de tipo bañado vinculados a los sistemas fluviales son causados mayoritariamente por el deterioro del hábitat, la contaminación, la regulación de los flujos hídricos y la extracción de agua. A su vez, la sobreexplotación de sus recursos naturales, la introducción de especies exóticas y las consecuencias del cambio climático, representan una amenaza para la biodiversidad que sostienen.

En cuanto a la diversidad biológica se debe tener en cuenta que una discriminación taxonómica más fina de los macroinvertebrados podría elevar seguramente los valores de los índices y evidenciarían diferencias más notables entre los ambientes remarcando también la estacionalidad climática entre períodos secos y húmedos. En estudios preliminares de invertebrados en la vegetación se hallaron diferencias significativas presentando los bañados del Cajaravillas y del Chubichaminí los mayores valores de riqueza (Altieri et al., 2019). Al igual que para otros ambientes de la zona pampeana (Rodrigues Capítulo et al., 2001; 2019; García et al., 2009; Juárez et al., 2016), la utilización de índices bióticos tales como el IBPAMP permitieron identificar diferencias entre los ambientes muestreados. En el caso del bañado del $\mathrm{A}^{\circ}$ Chubichaminí, donde solo existe una ganadería extensiva, el valor del éste índice 
promedio fue cercano a $8, y$, si se complementa con los valores estimados para el escore PBMWP para este sitio, que supera con el DS los 90 puntos, nos está indicando una calidad del agua aceptable; en cambio para los bañados relacionados con el $\mathrm{A}^{\circ}$ Cajaravillas y Carnaval los índices nos muestran una situación de calidad dudosa, y un estado "muy crítico con aguas muy contaminadas" para el bañado Del Gato donde la actividad antrópica es más intensa (Tabla 1). Cabe mencionar que, si bien los índices bióticos utilizados aquí fueron diseñados en su origen para sistemas lóticos propiamente dichos, su aplicación en estos humedales de desborde fluvial (generalmente interconectados) ha resultado muy interesante ya que ha permitido aproximar a una diferenciación del estado ecológico de los mismos complementándolo con las variables geomorfológicas y químicas la disponibles. Es probable que si se consideraran a los macroinvertebrados incluidos en la vegetación flotante y palustre, no considerados específicamente en esta contribución (dado que solo fueron consideradas las muestras extraídas con draga Ekman), estos valores se incrementarían significativamente dado que en estudios previos para los mismos sitios donde las macrófitas fueron incluidas los autores observaron otros organismos sensibles tal es el caso de odonatos Lestidae, o almejas nacaríferas como $D$. delodontus, (Rodrigues Capítulo et al., 2018; Altieri et al., 2019). Cabe mencionar que, en un análisis comparativo de utilización de los índices bióticos para ríos de Santiago del Estero, los valores de estos índices así como los taxa intervinientes se duplicaban al considerar los invertebrados de la vegetación (Leiva et al., 2020).

La mayor cobertura de la vegetación observada trabajos previos en el bañado del arroyo Del Gato (Figura 1 d), según Zanotti y Gómez (2020) y Rodrigues Capítulo et al., (2018), fue significativa y podría ser a simple vista un beneficio ecosistémico importante al proporcionar refugios para albergar a una alta diversidad de macroinvertebrados. Sin embargo, el efecto negativo de sustancias exógenas proveniente del sector hortícola y urbano, junto a la baja concentración de $\mathrm{O}_{2}$, en parte por la acumulación de MO alóctona y autóctona (Cortese et al., 2018), impedirían el desarrollo de poblaciones de macroinvertebrados más sensibles como las almejas nacaríferas ( $D$. delodontus), odonatos Lestidae, efemerópteros Baetidae y cangrejos Trichodactylidae.

La riqueza de especies no solo está controlada por factores locales sino también por procesos que se desarrollan a escalas regionales, como la dispersión y los eventos históricos. Los ensambles de especies con baja dispersión, como los oligoquetos, generalmente están influenciados por la composición de los sitios vecinos y solo suelen ser diferentes a lo largo de gradientes extensos (Behrend et al., 2012). En algunas especies de este grupo la colonización local está relacionada con su plasticidad, su capacidad de reproducción asexual y de producir cocones o quistes enterrados en sedimentos que pueden tolerar la adversidad impuesta por eventos catastróficos (por ejemplo, inundaciones y sequías). Por lo tanto, la dispersión puede actuar como una fuerza estructurante que conduce a un flujo constante de propágulos donde los sitios estrechamente conectados albergan conjuntos más similares que los sitios más alejados (Behrend et al., 2012). $\mathrm{Si}$ bien en este estudio se hallaron diferencias en los ensambles de oligoquetos en los cuatro arroyos analizados, estas diferencias pudieron deberse principalmente a los diferentes usos del suelo y al impacto de la actividad humana. En la distribución de las especies son particularmente importantes las características químicas del agua y del sustrato (Schenková et al., 2016) así como también el desarrollo de algas y vegetación acuática (Kang-Jieh \& Shao-Pin, 2008). La presencia de Limnodrilus hoffmeisteri Claparède, 1862, Tubifex tubifex (Müller, 1774) y Aulodrilus pigueti Kowalewski, 1914 suele asociarse con sedimentos limososarcillosos, con un alto contenido de materia orgánica y aumento en la conductividad (Ezcurra de Drago et al., 2007; Kang-Jieh \& Shao-Pin, 2008). Solo unas pocas especies pueden 
soportar las condiciones estresantes resultantes de un aporte excesivo de materia orgánica, dando lugar a comunidades simplificadas conformadas por un pequeño número de especies tolerantes, como por ejemplo $L$. hoffmeisteri, especie dominante en sitios con baja calidad del agua debido a la degradación física del hábitat y a las actividades antrópicas (Arslan et al., 2016). Por su parte, las especies del género Dero Oken, 1815 son indicadores de condiciones ambientales relacionadas con la contaminación orgánica y bajos niveles de concentración de oxígeno, pero además se asocian a la productividad de fitoplancton, que es su principal fuente de alimento (Behrend et al., 2012). A partir de los resultados obtenidos en este estudio, los Chironomidae no han mostrado variación significativa a lo largo del gradiente prebañado, bañado y postbañado. La riqueza (S) anual de Chironomidae observada en nuestro estudio fue más alta en los arroyos con menor grado de perturbación, mientras que los valores de diversidad $\left(\mathrm{H}^{\prime}\right)$ no mostraron una disminución en aquellos arroyos con mayor perturbación. Inclusive, los índices de equitabilidad y dominancia resultaron congruentes entre sí, donde expresan un ensamble con pocos individuos por especie, sin ninguna especie dominante. En donde sí existieron diferencias, fue entre los arroyos con menor y mayor perturbación, tanto en la cantidad de individuos como en la composición de especies. El índice de distancia taxonómica presentó su valor más bajo en el $\mathrm{A}^{\circ}$ Del Gato, sugiriendo un deterioro de las funciones del ecosistema y cambios ambientales (Clarke \& Warwick 1998, 1999).

La diversidad hallada fue superior a la de estudios previos en el área de interés donde se han utilizado las mismas técnicas de muestreo; de esta manera en arroyos urbanizados se contabilizaron cinco géneros/ morfotipos pertenecientes a la subfamilia Chironominae (Ocon \& Rodrígues Capítulo, 2004) y nueve especies repartidas en las subfamilias Chironominae y Orthocladiinae (Cortelezzi et al., 2011). En todos los trabajos citados y en este estudio se observa un predominio de Chironominae sobre el resto de las subfamilias. Dicha subfamilia es el grupo dominante en los ambientes de llanura tropical y subtropical, prefiriendo las aguas de menor velocidad de corriente o estancadas y en un rango de temperaturas medias a altas. Entre sus preferencias alimentarias predominan las formas detritívoras (Paggi, 2009). Las características ecológicas de Chironominae pueden ser muy amplias en algunos géneros $y$ especies, encontrando especies muy tolerantes a ambientes contaminados como Chironomus calligraphus, donde se ha estudiado en detalle su bionomía (Spies et al. 2002; Zilli et al., 2008). Las mayores densidades fueron observadas dentro de la subfamilia Chironominae, para los géneros Rheotanytarsus, Chironomus, Dicrotendipes Kieffer, Parachironomus y Polypedilum. Para el caso del arroyo del Gato, el cual presenta las características de un ambiente más impactado, los géneros Chironomus, Polypedilum y Parachironomus fueron los que presentaron mayor abundancia. Estos géneros que también estuvieron presentes en el resto de los arroyos están representados por más de una especie en la región. Como se expresó anteriormente, la distinción entre las especies a través de las larvas generalmente es inviable, ya que en el estudio se contabilizaron las larvas de todos los estadios, mientras que la mayor diferenciación se realiza a través del 4to estadio. De esta manera, es probable que exista una diferenciación entre las especies (o en las proporciones en que aparecen) de los diferentes bañados. Para el caso de Chironomus, a partir de las larvas de 4to estadio recolectadas en el arroyo del Gato, se pudo corroborar que la mayor parte corresponden a C. calligraphus, lo cual está en concordancia con lo que expresa Spies et al. (2002) acerca de la tolerancia y preferencia de esta especie por ambientes con evidente impacto antrópico. Por otro lado, especies de Polypedilum y Parachironomus también han sido reportadas previamente como tolerantes a condiciones de alto impacto antropogénico (Helson et al., 2006; Moreno \& 
Callisto, 2006). Sin embargo, en nuestro estudio no se ha podido realizar la diferenciación a nivel específica, lo que hubiese permitido corroborar si existen diferencias en cuanto a la relación de cada especie de estos géneros con el grado de impacto del ambiente.

La necesidad de contar con más estudios en estos temas es evidente, si bien se ha comenzado con nuevas líneas de investigación, como es el caso de la técnica de exuvias pupales donde se analizó la estructura y composición del ensamblaje de quironómidos y sus patrones de emergencia en relación con las variables ambientales. Dicha técnica permitió obtener un mayor número de géneros y especies para la región, y además se estudiaron las variables ambientales que estructuraron los patrones de emergencia (Zanotto Arpellino et al., "en prensa").

Las proporciones de GFA no mostraron un patrón claro que permita diferenciar arroyos con diferente uso del suelo. En el arroyo Del Gato se observó una alta proporción de depredadores y en el Carnaval de raspadores, en coincidencia con lo hallado por Solís et al. (2019), para arroyos con usos del suelo agrícola. Sin embargo, estos arroyos no estuvieron caracterizados por el mismo GFA dominante, correspondiendo la mayor proporción a colectores-recolectores en el primero y colectores filtradores en el segundo. Entre los arroyos con uso de suelo ganadero también hubo diferencias en el ensamble de GFA, si bien ambos tuvieron una importante presencia de colectores-recolectores, el principal grupo para el arroyo Chubichaminí fue el de los filtradores, mientras que para Cajaravillas fue el de los fragmentadores.

La alta densidad de bivalvos presentes en los arroyos Carnaval y Chubichaminí determina la elevada proporción de colectores-filtradores observados. Esto se relaciona estrechamente con la presencia de materia orgánica particulada fina en suspensión. En el primer caso esto puede relacionarse con el tipo de agricultura practicada en la zona (cultivos de soja), mientras en el otro arroyo puede deberse a la re-suspensión de partículas debida al pisoteo del ganado.

El estudio llevado a cabo en el presente trabajo permitió observar, a partir de los macroinvertebrados en general $y$, de los oligoquetos y quironómidos en particular, como sus ensambles se ven influenciados por el uso del suelo y las consecuentes características ambientales que modelan su estructura y función.

\section{AGRADECIMIENTOS}

El trabajo realizado fue financiado por los siguientes Proyectos: Consejo Nacional de Investigaciones Científicas y Técnicas (CONICET): PI UE-22920160100049CO; PIP: 2013-0570; Fondo Nacional de Ciencia y Tecnología (FONCYT): PICT 2014-1342; UNLP -FCNyM: 2014 11/ N 0738: 2018 11/ N 0869.

MD, AS, LRC y JPZA agradecen a la Universidad Nacional de La Plata (FCNyM, 11 / N785 y 11/ N914) y el Consejo Nacional de Investigaciones Científicas y Técnicas (PIP 1010), por parte del apoyo para realizar este estudio.

Se agradece especialmente a los miembros de la CPA CONICET, Profesionales Jorge L. Donadelli y Máximo A. Simonetti por su apoyo en los análisis de laboratorio y al Técnico Principal Roberto F. Jensen por su colaboración en los muestreos de campo.

\section{REFERENCIAS}

Alba Tercedor, J., Jáimez-Cuéllar, P., Álvarez, M., Avilés, J., Bonada, N., Casas, J., Mellado, A., Ortega, M., Pardo, I., Prat, N., Rieradevall, M., Robles, S., Sáinz-Cantero, C. E., SánchezOrtega, A., Suárez, M. L., Toro, M., VidalAbarca, M. R., Vivas, S. y Zamora-Muñoz, C., (2002). Caracterización del estado ecológico de ríos mediterráneos ibéricos mediante el índice IBMWP (antes BMWP'). Limnetica, 21(34), 175-185. 
Tomé, Asociación de Ciencias Naturales del Litoral: Colección Climax No. 6. Capítulo, A. (2019). Análisis preliminar de ensamble de macroinvertebrados en bañados de desborde fluvial del área pampeana. En: Cortelezzi, A., Entraigas, I. y Grosman F (Eds). Encuentro de saberes para la gestión responsable de ecosistemas acuáticos pampeanos (pp. 256258). Azul: Universidad Nacional del Centro de la Provincia de Buenos Aires.

APHA (1998). Standard methods for the examination of water and wastewater. Washington, DC: American Public Health Associatio,.

Armendáriz, L. C., Rodrigues Capítulo, A. \& Ambrosio, E. S. (2011). Relationships between the spatial distribution of oligochaetes (Annelida, Clitellata) and environmental variables in a temperate estuary system of South America (Río de la Plata, Argentina). New Zealand Journal of Marine and Freshwater Research, 45, 263-279.

Armendáriz, L. C., Cortese, B., Rodríguez, M. \& Rodrigues Capítulo, A. (2017). Ecosystem services of runoff marshes in urban lowland basins: proposals for their management and conservation. Knowledge and Management of Aquatic Ecosystems, 418, 32.

Armitage, P. D., Moss, D., Wright, J. F. \& Furse, M. T.(1983). The performance of a new biological water quality score system based on macroinvertebrates over a wide range of unpolluted running-water sites. Water Research, 17, 333-34.

Arslan, N., Salur, A., Kalyoncu H., Mercan D., Odabasi, D. A. \& Barısık, B. (2016). The use of BMWP and ASPT indices for evaluation of water quality according to macroinvertebrates in Kucuk Menderes River (Turkey). Biologia, Section Zoology.

Behrend, R. D. L., Takeda, A. M., Gomes, L. C. \& Fernandes, (2012). Using oligochaeta assemblages as an indicator of environmental changes. Brazilian Journal of Biology, 72, 873-884.

Brinkhurst, R. O. y Marchese, M. R. (1992). Guía para la identificación de oligoquetos acuáticos continentales de Sud y Centroamérica. Santo
Barbour, M. T., Gerritsen, J., Snyder, B. D. \& Stribling, J.B. (1999). Rapid bioassessment protocols for use in streams and wadeable rivers: periphyton, benthic macroinvertebrates and fish. EPA/841-B-99-002. Washington, D.C.: U.S. EPA, Office of Water.

Bunn, S., Davies, P. \& Mosisch, T. (1999). Ecosystem measures of river health and their response to riparian and catchment degradation. Freshwater Biology, 41, 333-345.

Clarke, K. R., \& Warwick, R. M. (1998). A taxonomic distinctness index and its statistical properties. Journal of Applied Ecology, 35, 523-531.

Clarke, K. R., \& Warwick, R. M. (1999). The taxonomic distinctness measure of biodiversity: Weighting of step lengths between hierarchical levels. Marine Ecology Progress Series, 184, 21-29.

Cortelezzi, A., Paggi, A. C., Rodríguez, M., \& Rodrigues Capítulo, A. (2011). Taxonomic and nontaxonomic responses to ecological changes in an urban lowland stream through the use of Chironomidae (Diptera) larvae. Science of the Total Environment, 409(7), 1344-1350.

Cortelezzi, A., Simoy, M.vV., Siri, A., Donato, M., Cepeda, R.vE., Marinelli C. B. \& Berkunsky, I. (2020). New insights on bioindicator value of Chironomids by using occupancy modelling. Ecological Indicators, 117, 106619.

Cortese, B, Simonetti, M, Jensen, R. F., Donadelli, J. y Rodrigues Capítulo, A. (2018). Estudio preliminar sobre la amortiguación de metales pesados en sedimentos de bañados de desborde fluvial pampeanos. Su evaluación mediante índices bióticos con macroinvertebrados. Biologia Acuática, 32, 115.

Cortese, B., Zanotto Arpellino J. P., Paggi, J. P. \& Rodrigues Capítulo, A. (2019). Chironomid genera distribution related to environmental characteristics of a highly impacted basin (Argentina, South America). Environmental 
Science and Pollution Research, 26,8087-8097.

Cummins, K. (1973). Trophic relations of aquatic insects. Annual Review in Entomology, 18, 183206.

Cummins, K., Merritt, R. \& Andrade, P. (2005). The use of invertebrate functional groups to characterize ecosystem attributes in selected streams and rivers in south Brazil. Studies on Neotropical Fauna and Environment, 40, 69-89.

Davidson, N. C. (2014). How much wetland has the world lost? Long-termand recent trends in global wetland area. Marine and Freshwater Research, 65(10), 936-941.

Domínguez, E. y Fernández, H. R. (Eds.). (2009). Macroinvertebrados bentónicos. Sudamericanos. Sistemática y biología. Tucumán: Fundación Miguel Lillo.

Epler, J. H. (2001). Identification manual for the larval Chironomidae (Diptera) of North and South Carolina: A guide to the taxonomy of the midges of the southeastern United States, including Florida. North Carolina Department of Environment and Natural Resources, Raleigh, NC, and St. Johns River Water Management District, Palatka, FL.

Ezcurra de Drago, I., Marchese, M. \& Montalto, L. 2007. Benthic Invertebrates. In: Iriondo, M. H., Paggi, J. C. \& Parma, M. J. (Eds.). The Middle Paraná River: Limnology of a Subtropical Wetland. (pp. 251-275). Verlag, Berlin Heidelberg New York: Springer.

Fenoglio, S., Bo, T., Agosta, P. \& Malacarne, G. (2005). Temporal and spatial patterns of coarse particulate organic matter and macroinvertebrate distribution in a Low-Order Apennine Stream. Journal of Freshwater Ecology, 20, 539-547.

Ferrington, L. C. (2008). Global diversity of nonbiting midges (Chironomidae; Insecta-Diptera) in freshwater. In: Balian, E.V., Lévéque, C., Segers, H. \& K. Martens (Eds.). Freshwater animal diversity assessment (pp. 447-455). Netherlands: Springer.
García, M. E., Rodrigues Capítulo, A. y Ferrari, L. (2009). El ensamble de invertebrados y la calidad del agua: indicadores taxonómicos y funcionales en arroyos pampeanos. Biología Acuática, 26, 109-120.

Gómez, N., Rodrigues Capítulo, A., Colautti, D., Mariñelarena, A., Magdalena, L., Cochero J., Armendáriz, L., Maroñas, M., Donadelli, J., Jensen, R., Garcia de Souza, J., Maiztegui, T., García, I., Sathicq, M. B., Suarez, J. C. y Cortese, B. (2016). La puesta en valor de los servicios ecosistémicos que ofrecen los arroyos de llanura como una medida de mitigación de las inundaciones: el caso del $A^{\circ}$ Del Gato en el Partido de La Plata. (39-52) En: Volpedo, A.V., de Cabo, L., Arreghini, S. y Fernández Cirelli, A. (Eds.). Ecología y manejo de ecosistemas acuáticos pampeanos. Buenos Aires: VIII EMEAP.

Gómez-Lemos, L., Cruz Castaño, N. y Duque Nivia, G. (2010). Composición y estructura del ensamblaje de crustáceos Brachyura de la plataforma continental de la Guajira Colombiana y su relación con la profundidad y las características del sedimento. Boletín de Investigaciones Mararinas y Costeras, 39(2), 359-379.

Hammer, Ø., Harper, D. A. T. \& Ryan, P. D. (2001). PAST: Paleontological Statistics Software Package for Education and Data Analysis. Palaeontologia Electronica, 4(1), 1-9.

Helson, J. E., Williams, D. D. \& Turner, D. (2006). Larval Chironomid community organization in four tropical rivers: human impacts and longitudinal zonation. Hydrobiologia, 559(1), 413-431.

International Commission on Zoological Nomenclature, (2007). Bulletin of Zoological Nomenclature. Opinion 2167.

Juárez, R., Crettaz-Minaglia, M. C., Aguer, I., Juárez, I., Gianello, D., Ávila, E. y Roldán C. (2016). Aplicación de índices bióticos de calidad de agua en cuatro arroyos de la cuenca del río Gualeguaychú (Entre Ríos, Argentina). Revista Intropica, 11, 35 - 46. 
Kang-Jieh, L. \& Shao-Pin, Y. (2008). The effect of organic pollution on the abundance and distribution of aquatic oligochaetes in an urban water basin, Taiwan. Hydrobiologia, 596, 213-223.

Krodkiewska, M. \& Michalik- Kucharz, A. (2009). The bottom Oligochaeta communities in sand pits of different trophic status in Upper Silesia (Southern Poland). Aquatic Ecology, 43, 437-444.

Lafont, M., Camus, J. C., Fournier A. \& Sourp, E. (2001). A practical concept for the ecological assessment of aquatic ecosystems: application on the River Dore in France. Aquatic Ecology, 35 , 195-205.

Leiva, M., Bonacina, E. y Diodato, L. (2020). Efecto del muestreo de diferentes hábitats sobre la aplicación de índices bióticos basados en Macroinvertebrados. Biología Acuática, 34, 1-12.

Lopretto, E. C. y Tell, G. (Dirs.) (1995). Ecosistemas de aguas continentales. Metodologías para su estudio. La Plata: Ediciones Sur.

Marchese, M. R. (2009). Annelida Oligochaeta. En: Domínguez, E. y Fernández, H.R. (Eds.). Macroinvertebrados bentónicos sudamericanos, sistemática y biología (pp. 551-565). Tucumán: Fundación Miguel Lillo.

Merritt, R. W. \& Cummins K. W. (1996). An introduction to the aquatic insects of North America. Kendal Hunt Publishing Co.

Merritt, R. W., Cummins, K. W. \& Berg, M. B. (2008). An introduction to the aquatic insects of North America. Dubuque: Kendall-Hunt.

Millennium Ecosystem Assessment (M.E.A.). (2005). Ecosystems and human well-being: synthesis. Washington, DC: Island Press.

Moreno, P. \& Callisto, M. (2006). Benthic macroinvertebrates in the watershed of an urban reservoir in southeastern Brazil. Hydrobiologia, 560, 311-321.
Neiff, J. J. (2001). Humedales de la Argentina: sinopsis, problemas y perspectivas futuras. En: Cirelli, A. F. (Ed.). El agua en Iberoamerica, funciones de los humedales, calidad de vida y agua segura (pp. 83-112). Argentina: CYTED.

Ocon, C. \& Rodrigues Capítulo, A. (2004). Presence and abundance of Ephemeroptera and other sensitive macroinvertebrates in relation with habitat conditions in pampean streams (Buenos Aires, Argentina). Archiv fuer Hydrobiologie, 159(4), 473-487.

Paggi, A. C. (1999). Los Chironomidae como indicadores de calidad de ambientes dulceacuícolas. Revista de la Sociedad Entomológica Argentina, 58(1-2), 202-207.

Paggi, A. C. (2009). Diptera. Chrionomidae. En: Domínguez, E. \& Fernández, H. R. (Eds.). Macroinvertebrados Bentónicos Sudamericanos. Sistemática y Biología (pp. 383-409). Tucumán: Fundación Miguel Lillo.

Pelegri, S. P. \& Blackburn, T. H. (1995). Effects of (Oligochaeta: Tubificidae) on $\mathrm{N}$-mineralization in freshwater sediments, measured with $15 \mathrm{~N}$ isotopes. Aquatic Microbial Ecology, 9,289-294.

Pinder, L. C. V. (1986). Biology of freshwater Chironomidae. Annual Review of Entomology, 31(1), 1-23.

Ramírez, A., \& Gutiérrez-Fonseca, P. E. (2014). Functional feeding groups of aquatic insect families in Latin America: a critical analysis and review of existing literature. Revista de Biología Tropical, 62, 155-167.

Ringuelet, R.A. (1962). Ecología acuática continental. Buenos Aires: Manuales de EUDEBA.

Rodrigues Capítulo, A. (1999). Los macroinvertebrados como indicadores de calidad de ambientes lóticos en el área pampeana. Revista de la Sociedad Entomológica. Argentina, 58, 208-217. 
Rodrigues Capítulo, A., Tangorra, M. \& Ocon, C. (2001). Use of Benthic macroinvertebrate to assess the biological status of pampean streams in Argentina. Aquatic Ecology, 35(2), 109-119.

Rodrigues Capítulo, A., Ocón, C. S. y Tangorra, M. (2004). Una visión bentónica de ríos y arroyos pampeanos. Biología Acuática, 21, 1-17.

Rodrigues Capítulo, A., Cortelezzi, A., Ocón, C., Spaccesi, F., Ambrosio, E. S. y López van Oosterom, V. (2006). Informe estudios línea de base y biomonitoreo - río Uruguay. Estado actual de las poblaciones de meso y macroinvertebrados y su relación con la posible contaminación producto de las industrias papeleras (Entre ríos, Argentina). ILPLA, FCNyMUNLP.

Rodrigues Capítulo, A., Cortelezzi, C. Ocón, F. Spaccesi, L. Armendáriz, A.C. Ferreira, V. López van Oosterom, E. S. Ambrosio, (2008). Bentos del tramo inferior del río Uruguay. Estado actual de las poblaciones de macro y meso invertebrados y su relación con la posible contaminación producto de las industrias papeleras (Entre Ríos, Argentina) (3 informes: 2007-2008). Convenio SAYDS (Secretaria de Ambiente y Desarrollo Sustentable de la Nación), Universidad Nacional de la PlataFCNyM-ILPLA).

Rodrigues Capítulo, A., Muñoz, I., Bonada, N., Gaudes, A. y Tomanova, S. (2009). Capítulo 14. La biota de los ríos: los invertebrados. En: Elosegui, A. y Sabater, S. (Eds). Conceptos y técnicas en ecología fluvial (pp. 285-301). Bilbao: Fundación BBVA.

Rodrigues Capítulo, A., Gómez, N., Giorgi, A., \& Feijoo, C. (2010). Global changes in pampean lowland streams (Argentina): implications for biodiversity and functioning. Hidrobiología, 657, 53-70.

Rodrigues Capítulo, A., Cortese, B., Armendáriz, L. C., Rodríguez, M., Rodrigues Capítulo, L., Ferreira, Ac., Altieri, P., Simonetti, M., Jensen, R., Gómez, N., (2018). ¿Resultan eficientes los bañados de desborde fluvial pampeanos en la mejora de la calidad del agua? Visión desde los invertebrados y las macrófitas. Libro de Resúmenes CAL VIII. UNLU.:143., 143. Lujan, Buenos Aires.

Rodrigues Capítulo, A., Armendáriz, L. C., Ocon, C. S., Paz, L. E., Altieri, P. D., Rodriguez, M., Cortese, B., Ferreira, A. C., Spaccesi, F. y Cortelezzi, A. (2019). Estado ecológico de los ambientes lóticos de la Ecorregión Pampeana basado en índices biológicos a partir de los ensambles de macroinvertebrados. XVII Congresso Brasileiro de Limnologia \& $2^{\circ}$ Congresso Ibero-Americano de Limnologia, Florianópolis, Santa Catarina.

Rodrigues Capítulo, L., Kruse, E. y Gómez, N., (2020). Los bañados de desborde fluvial: una mirada desde la geohidrología. Biología Acuática, 35.

Rodríguez, P. \& Reynoldson, T. B. (2011). The pollution biology of aquatic Oligochaetes. New York: Springer.

Schmelz, R. M. \& Collado, R. (2010). A guide to European terrestrial and freshwater species of Enchytraeidae (Oligochaeta). Soil Organisms, $82(1), 1-176$.

Schenková, J., Bílková, M. \& Horsák, M. (2016). The response of Clitellata (Annelida) to environmental gradients inspring fens. Limnologica, 57, 73-82.

Silva, F. L., Pinho, L. C., Wiedenbrug, S., Dantas, G. P. S., Siri, A., Andersen, T., \& Trivinho-Strixino, S. (2018). Family Chironomidae. In: Hamada, N., Thorp, J. H. \& Rogers, D.C (Eds.). Thorp and Covich's Freshwater Invertebrates (pp. 661-700). New York: Academic Press.

Spies, M., Sublette, J. E., Sublette, M. F., Wülker, W. F., Martin, J., Hille, A., Miller, M. A. \& Witt, K. (2002). Pan-American Chironomus calligraphus Goeldi, 1905 (Diptera, Chironomidae): Species or Complex? Evidence from External Morphology, Karyology and DNA Sequencing. Aquatic Insects, 24(2), 91-113. 
ter Braak, C.J. F. (1986). Canonical correspondence analysis: a new eigenvector technique for multivariate direct gradient analysis. Ecology, 67,1167-1179.

ter Braak C. F. J. \& Verdonschot, P. F. M. (1995). Canonical correspondence analysis and related multivariate analysis in aquatic ecology. Aquatic Sciences, 57(3), 255-289

U.S. EPA. (2015). Connectivity of streams and wetlands to downstream waters: A review and synthesis of the scientific evidence (Final Report). Washington, DC: U.S. Environmental Protection Agency.

Vorobyev, D. S., Frank, Y. A., Lushnikov, S. V., Zaloznyi, N. A. \& Noskov, Y. A. (2010). Use of Limnodrilus hoffmeisteri (Tubificidae, Oligochaeta) for purification of bottom sediments from oil and oil products. Sibirskii Ekologicheskii Zhurnal,1,21-27.

Wetzel, M. J., Fend, S., Coates, K. A., Kathman, R. D. \& Gelder, S. R. (2006). Taxonomy, systematics and ecology of the aquatic Oligochaeta and Branchiobdellida (Annelida, Clitellata) of North America, with emphasis of the fauna occurring in Florida: A workbook. Florida: Departament of Environmental Protection - FDEP.
Zanotti, C. y Gómez, N. (2019). Caracterización de bañados de desborde fluvial a partir de inventarios florísticos. En: Cortelezzi, A., Entraigas, I., Grosman, F. y Masson, I. (Eds). Encuentro de saberes para la gestión responsable de ecosistemas acuáticos pampeanos (pp. 116118). Azul: Universidad Nacional del Centro de la Provincia de Buenos Aires.

Zanotto Arpellino, J. P., Rodríguez Catanzaro, L. N. S, Montalto, L., Siri, A. \& Donato, M. (In press). Diversity, phenology and voltinism of Chironomidae (Diptera). neotropical streams as a study model. Anais da Academia Brasileira de Ciências.

Zilli, F. L., Montalto, L., Marchese, M. R. (2008) a. Benthic invertebrate assemblages and functional feeding groups in the Paraná River floodplain (Argentina). Limnologica, 38, 159-171.

Zilli, F., Montalto, L., Paggi, A.C., \& Marchese, M. (2008)b. Biometry and life cycle of Chironomus calligraphus Goeldi 1905 (Diptera: Chironomidae) in laboratory conditions. Interciencia, 33,767-770.

Zornoza, A. (2015). Metodología para la búsqueda de microorganismos bioindicadores en fangos activos: la desesperación en la búsqueda del modelo. Curso teórico-práctico "El papel de los protistas en los procesos de depuración de las aguas residuales". Sevilla, España. 